\title{
Les humanistes français, le roi et le tyran. Débats autour du tyrannicide au sein du milieu humaniste français, $1^{\text {ère }}$ moitié du XV $\mathrm{XV}^{\mathrm{e}}$ siècle
}

French humanists, the king and the tyrant. Debates on tyrannicide in the French humanist milieu, 1st half of the 15th century

\section{Lucie Jollivet}

\section{OpenEdition}

Journals

Édition électronique

URL : http://journals.openedition.org/medievalista/1641

DOI : 10.4000/medievalista.1641

ISSN : 1646-740X

Éditeur

Instituto de Estudos Medievais - FCSH-UNL

Édition imprimée

Date de publication : 1 janvier 2018

Référence électronique

Lucie Jollivet, « Les humanistes français, le roi et le tyran. Débats autour du tyrannicide au sein du milieu humaniste français, 1 ère moitié du XVe siècle », Medievalista [En ligne], 23 | 2018, mis en ligne le 07 mai 2018, consulté le 20 avril 2019. URL : http://journals.openedition.org/medievalista/1641 ; DOI : 10.4000/medievalista.1641

Ce document a été généré automatiquement le 20 avril 2019.

(c) IEM 


\section{Les humanistes français, le roi et le tyran. Débats autour du tyrannicide au sein du milieu humaniste français, $1^{\text {ère }}$ moitié $d u X V^{\mathrm{e}}$ siècle}

French humanists, the king and the tyrant. Debates on tyrannicide in the French humanist milieu, 1st half of the 15th century

Lucie Jollivet

\section{NOTE DE L'ÉDITEUR}

Data recepção do artigo / Received for publication: 16-03-2017

Data aceitação do artigo / Accepted in revised form: 20-10-2017

Les humanistes français, le roi et le tyran. Débats autour du tyrannicide au sein du milieu humaniste

français, $1^{\text {ère }}$ moitié du XV siècle / French humanists, the king and the tyrant. Debates on tyrannicide in the French humanist milieu, 1st half of the 15th century

Lucie Jollivet

1 Le 23 novembre 1407, le meurtre avec préméditation de Louis d'Orléans, frère du roi Charles VI, par les sbires de leur cousin Jean de Bourgogne, remet la question du tyran et du tyrannicide au centre des débats entre intellectuels ${ }^{1}$. En effet, après avoir avoué être le commanditaire du crime, Jean sans Peur revient sur ses déclarations et demande à une équipe de théologiens, dirigée par le normand Jean Petit, de préparer sa défense ${ }^{2}$. Cette justification est exposée au public en 1408 et repose sur un argument repris au juristeconsulte Cassius: vim vi repellere licet. Louis aurait été un tyran, il aurait donc été à la fois licite et juste de le supprimer. L'actualité du crime rend la proposition de Jean Petit 
d'autant plus choquante que la justice royale se trouve paralysée face au refus de l'aveu. Devant l'incapacité de la justice publique à réagir, la famille et les proches du défunt n'ont plus d'autre choix que la vengeance pour d'obtenir réparation. Le milieu humaniste français, constitué d'humanistes avérés et reconnus, tels Nicolas de Clamanges, Laurent de Premierfait et Alain Chartier, et d'universitaires tentés par ce nouveau mouvement intellectuel comme Pierre d'Ailly et Jean Gerson ${ }^{3}$, réactivent une ancienne polémique en l'adaptant au contexte: Qu'est-ce qu'un tyran? Quelle est la différence entre le tyran et le roi légitime? Est-il licite de tuer le tyran pour sauver le roi? Il faut impérativement trouver la réponse la plus juste à ces questions car la sauegarde du royaume de France est en jeu. C'est cette réflexion globale autour du Bien Commun qui unit les auteurs, par delà la distance géographique ou générationnelle.

2 Nicolas et Laurent, tout d'abord, sont presque du même âge ${ }^{4}$ et participent à la fois aux cercles humanistes avignonnais et parisien. Ils ont en commun une verdeur de ton qui tranche avec la prudence d'un grand nombre de leurs contemporains. Alain Chartier, quant à lui, appartient à la $2^{\mathrm{e}}$ génération d'humanistes: c'est un laïc, fidèle serviteur du dauphin puis du roi Charles VII et fervent admirateur de Clamanges et sans doute de Premierfait, dont il ne peut méconnaître les traductions, notamment celles du De Casibus virorum illustrium et du Decameron de Boccace, achevées respectivement en 1409 et en 1414 ${ }^{5}$. Nicolas de Clamanges est très prolixe tout au long de sa vie mais ce sont deux œuvres fondamentales qui retiendront plus particulièrement notre attention: son traité De lapsu et reparatione justitie rédigé au début des années 1420 , ainsi que ce qu'il considérait comme son œuvre maîtresse, commencée sans doute dès le début du XV siècle mais demeurée inachevée et encore inédite à ce jour, l'Expositio super quadraginta septem capitula Isaye. Enfin, le poème en latin Ad detestationem belli gallici d'Alain Chartier, certainement composé juste avant 1419, nous permettra de mesurer l'impact des travaux de ses prédécesseurs, donc de souligner la circulation des idées dans le cercle humaniste français malgré un contexte défavorable. Au fil de cette étude, il apparaîtra qu’à la faveur de la polémique autour du tyrannicide, les œuvres des trois humanistes dévoilent leur idéal partagé d'un roi légitime capable de ramener la justice et la paix dans le royaume.

\section{Le tyrannicide? Une question d'une brûlante actualité}

"Il n'est sacrifice tant agreable a Dieu comme le sang d'un tirant et mauvaiz prince"'. Laurent de Premierfait, qui traduit ici littéralement la célèbre phrase de Boccace, s'étonne-t-il de l'entendre, le 8 mars 1408, dans la bouche du défenseur duc de Bourgogne, le théologien Jean Petit?? Ce dernier la brandit parmi d'autres auctoritates, tel le jurisconsulte Cassius $^{8}$, qui affirmait Vim vi repellere licet, pour justifier le meurtre odieux de Louis d'Orléans'. Le tyrannicide est en effet une question d'une brûlante actualité, comme le prouve le débat qui fait rage en Italie durant la $2^{\text {nde }}$ moitié du XIV siècle.

4 En 1400, l'humaniste florentin Coluccio Salutati, qui lutte alors contre la tyrannie de Gian Galeazzo Visconti, achève son traité De tyranno, dans lequel il admet l'axiome vim vi mais reste prudent lorsqu'il s'agit de légitimer le tyrannicide. Pour lui, Dante a eu raison de placer Cassius et Brutus en Enfer car le tyran ne peut être tué que sur ordre de l'Etat et dans le cadre strict de la loi. Or, César exerçait légalement le pouvoir... Jean Petit ne peut ignorer cet ouvrage car, s'il n'appartient pas lui-même au cercle humaniste, il en a pourtant côtoyé certains membres. La majeure de sa justification repose en outre sur la fameuse citation de saint Paul, radix omnium malorum est cupiditas, que Pierre d'Ailly avait 
placé au cœur de son accusation, victorieuse, contre le chancelier de l'Université de Paris, Jean Blanchard, en $1385^{10}$. Provocation?

5 L'ardeur combative de Jean Petit n'est pas une nouveauté en 1408, elle s'illustre en effet dès 1398, dans la lutte contre le schisme. Alors porte-parole de l'Université de Paris, il prend clairement position dans ses discours pour l'abdication simultanée des deux papes et soutient aux côtés de Simon de Cramaud que la soustraction d'obédience est la meilleure solution pour amener Benoît XIII à céder. Or, le milieu humaniste est bien plus indulgent à l'égard du pape, voire lui est personnellement attaché, comme Nicolas de Clamanges parti en 1394 à Avignon pour y devenir son secrétaire. D'Ailly et Gerson ne cachent pas leur hostilité face à la soustraction d'obédience et c'est finalement Louis d'Orléans, le frère du roi, qui convainc ce dernier de se réconcilier avec Benoît XIII et d'accepter la restitution d'obédience en 1403. D'ailleurs, cette décision est officiellement prononcée le 30 mai 1403 par nul autre que Pierre d'Ailly.

6 Mais Jean Petit ne désarme pas et en 1406, lors de l'assemblée du clergé, il mène l'attaque contre le pape en demandant une nouvelle soustraction d'obédience. En 1407, il fait partie, comme Pierre d'Ailly, Jean Gerson ou Jacques de Nouvion ${ }^{11}$, de l'ambassade de 36 membres qui se rend à Avignon, Marseille puis Gênes et Rome pour négocier la double cession avec Benoît XIII et Grégoire XII. Mais les divisions se font rapidement jour entre les soutiens du pape et les intransigeants, dont Petit et Cramaud font partie ${ }^{12}$. Jacques de Nouvion, proche de Louis d'Orléans, qualifie d'ailleurs le discours de Jean Petit à Rome de diffusius, "dispersé"13, critique qui peut aussi bien avoir une réalité stylistique, Nouvion appartient au cercle humaniste et on imagine que son jugement à l'égard de la prose de ses collègues peut être sévère, que révéler une mesquinerie personnelle face au discours d'un théologien appartenant au camp opposé.

7 Devant les atermoiements de Benoît XIII, certains ambassadeurs pensent que la nouvelle de la soustraction d'obédience, décidée par l'université parisienne, pourrait lui extorquer les garanties recherchées. Gerson, d'Ailly et Nouvion s'y refusent et parviennent à convaincre leurs collègues. On ne saurait douter qu'ils se font l'écho ici des conseils de Louis d'Orléans, qui a toujours ménagé le souverain pontife. Jean Petit proteste énergiquement, sans effet ${ }^{14}$. Dans sa Justification, il montre que ce souvenir est encore amer: il reproche nommément à Jacques de Nouvion, Jean Gerson et Pierre d'Ailly d'avoir contraint les ambassadeurs à garder le secret sur la $2^{\text {ème }}$ soustraction d'obédience. En outre, il accuse Nouvion d'être revenu auprès du duc d'Orléans afin de lui faire part de l'entente nouée entre les deux papes pour faire échouer les négociations. De là à rendre Nouvion et ses amis en partie responsables de l'échec de la résolution du schisme, il n'y a qu'un pas, vite franchi par le théologien normand ${ }^{15}$.

8 On voit donc que Jean Petit, devenu un protégé du duc de Bourgogne après 1395, a toutes les raisons, personnelles et professionnelles, pour s'attaquer en 1408 à la mémoire de Louis d'Orléans et à ceux qui voudraient la défendre ${ }^{16}$. Cependant, pour les humanistes français, la réalité du crime prend le pas sur les débats oratoires: les circonstances de l'acte, la diffamation de la victime et les conséquences de la justification sont autant de péchés et de violations du principe de justice.

9 Jean de Montreuil, dans une lettre adressée à Nicolas de Clamanges, entre novembre 1416 et avril 1417, se demande comment il a été possible de massacrer le frère du roi en pleine rue et comment ses assassins ont pu rester impunis, alors qu'il devait y avoir de nombreux témoins ${ }^{17}$. Son ami lui adresse une réponse dans l'Expositio super Isayam ${ }^{18}$ : "Je 
me demande ce qu'on peut attendre d'une justice ensevelie, quand les juges détournent leur regard des voleurs et des homicides officiels, pour le porter sur d'autres?".

La question est de pure forme: l'audace d'une embuscade tendue dans un quartier populeux de Paris, impliquant la présence de nombreux témoins potentiels ne laisse toujours pas d'étonner, plusieurs années après son déroulement ${ }^{19}$. Chartier, dans l'Ad detestationem, déplore: "les crimes les plus extrêmes [qui] se montrent [...] en public" ${ }^{20}$.

11 Le meurtre public entache la mémoire de la victime et couvre ses proches de honte. En outre, la blessure faite à l'honneur et à l'amour-propre est rendue encore plus cuisante par le déni de justice, appuyé sur la diffamation ${ }^{21}$. L'impunité dont a bénéficié Jean sans Peur a ensuite servi de précédent pour la légitimation des massacres suivants ${ }^{22}$.

12 Enfin, la Justification a été soutenue en public, comme le rappelle Chartier ${ }^{23}$. Comment s'étonner alors qu'une lutte fratricide déchire le royaume, quand la honte est infligée officiellement? S'inspire-t-il de Clamanges qui, dans l'Expositio fait clairement référence à la séance publique du 8 mars $1408^{24}$ quand il évoque: "la flagornerie ou l'injustice spontanée, captieuse et perverse de scribes ou de princes pernicieux habitués à se mêler de la loi ${ }^{25},[. .$.$] "les simples et les incultes se laissent [ainsi] séduire par les sophismes$ astucieux"26.

13 A cause de l'accusation de tyrannie portée contre Louis d'Orléans, le défunt est privé de la reconnaissance du statut légal de victime, sa famille et ses proches n'ont plus d'autre choix que la vengeance pour faire valoir leur droit. La justice sort alors du cadre public pour revenir dans le domaine privé et rappeler les pires vendettas du haut Moyen-Age. Pour les membres du cercle, la Justification est le scandalon, la pierre qui fait basculer la société dans la sédition:

“Là, [à Paris] tous les scandales furent provoqués et éclatèrent [...] N'est-ce pas là que les guerres civiles entre les princes prennent leur origine, suscitées par des paroles à double tranchant qui mirent le feu aux poudres et qui, en infligeant des plaies inouïes, hâtèrent le royaume vers sa fin universelle?" ${ }^{27}$.

14 Les humanistes s'indignent de la légitimation de l'assassinat, permise par une fallacieuse accusation de tyrannie. La Justification constitue pour la société chrétienne un piège qui l'éloigne toujours plus de la voie du salut. D'ailleurs, l'histoire offre aux lecteurs des exemples d'actes odieux qui entraînent toute une société dans le malheur. Les troubles ayant opposé, à Rome, Sylla et Marius, en 88-87 puis en 82 avant notre ère, semblent avoir particulièrement inspiré Laurent de Premierfait et Alain Chartier. Cet exemple est loin d'être anodin, puisqu'il fait référence à l'opposition entre les populares et les optimates, à l'origine des guerres civiles qui auront finalement raison de la République romaine.

15 La traduction de l'ouvrage de Boccace De casibus virorum offre à Laurent le moyen de dénoncer vigoureusement le pouvoir, avec une véhémence qui rappelle celle de Clamanges. Le passage du latin à la langue vulgaire est pour l'humaniste une occasion de développer certains passages, ce qui nous renseigne notamment sur sa conception de la souveraineté. Ainsi, les épisodes des guerres civiles romaines constituent pour l'auteur un sujet de choix ${ }^{28}$. Carla Bozzolo ${ }^{29}$ note que Laurent puise dans Lucain pour leur description; ce même choix sera fait par Chartier, mais avec un apport original, nous y reviendrons bientôt. L'apparition de Pompée sur la scène politique romaine, puis son affrontement final avec César permettent à Laurent de dresser un parallèle saisissant avec sa propre époque. Il évoque tout d'abord: "la cité de romme perillée et detruite par les dommageuses dissensions et guerres civiles qui longuement furent contre Marius et Silla, [...] enflammez de envie l'un contre l'autre" ${ }^{30}$. Comment ne pas penser à la jalousie 
que ressentaient l'un envers l'autre Louis d'Orléans et Jean de Bourgogne, alors qu'ils s'affrontaient pour contrôler le roi Charles VI? De même, l'évocation du corps mutilé de Pompée sur le champ de bataille ${ }^{31}$ rappelle celui de Louis d'Orléans, retrouvé étendu à terre, le crâne fendu et le poing tranché. La dépouille de Pompée est privée de funérailles dignes de son rang, des pleurs et des louanges qu'il aurait mérités. Si Louis d'Orléans reçoit des obsèques royales, autour du cercueil on ne voit ni le roi ni ses fils, pas plus que la veuve, exilée à Château-Thierry et qui ignore encore la mort de son mari ${ }^{32}$. En outre, la joie du peuple contraste avec la tristesse des proches et le refus de l'aveu du commanditaire vient troubler le repos du défunt. A Rome, les parents et amis du grand homme vaincu jurent alors de "faire la vengeance de Pompée" 33 , comme Valentine Visconti et ses enfants, puis ses soutiens, derrière le clan armagnac. Enfin, la description de la guerre civile qui en découle est la vivante évocation du conflit opposant Armagnacs et Bourguignons après 1408: "En celle maudite et cruelle bataille entretoient les peres leurs enfans, les enfans leurs peres, les parens leurs amis et les voysins l'un l'autre [...]" ${ }^{34}$. Mais l'auteur se réserve pour plus tard une description plus minutieuse des faits: "je di que chose trop longue seroit ja [...] de maintenant escrire [...]". Toujours est-il que le meurtre d'un homme d'Etat provoque nécessairement une sédition affectant toute le corps social.

Quelques années plus tard, le conflit n'est toujours pas résolu. Dans son poème dénonçant les horreurs d'une guerre "intestine", Alain Chartier utilise à son tour les sources antiques pour mieux éclairer le terrible présent et revient sur la guerre civile entre Sylla et Marius: "Cette rage toute sillanienne s'empare de Rome, devant laquelle Sylla le furieux se pare du titre d'artisan de la paix, après que Rome ait gardé le silence lors du massacre public de Marius" 35 .

En 88 av. J.-C. Sylla marche sur Rome avec ses troupes, ce qui est un acte illégal. Il élimine ensuite ses opposants et fait voter aux sénateurs apeurés un senatus-consulte mettant tous ses adversaires hors-la-loi. La paix est alors acquise, comme le dit Chartier, au prix de la terreur. Sylla prendrait ensuite, en flagrante opposition avec la réalité de la situation, le titre de pacis auctorem, artisan de la paix. Cette expression se trouve déjà dans les Histoires de C. Velleius Paterculus ${ }^{36}$, historien romain qui est le seul à l'avoir utilisée en évoquant Sylla. L'humaniste complète donc habilement Lucain grâce à cette source moins connue.

Contrairement à Laurent, qui en suivant Boccace soulignait la déchéance morale de Marius, à l'origine de son échec et de sa mort ignominieuse, Alain Chartier présente Marius comme une victime de la folie meurtrière de Sylla, mais la guerre civile entre César et Pompée fait également l'objet d'une description pathétique: "les yeux portant leur regard sur les champs baignés du sang romain pleurent abondamment" ${ }^{37}$. Nous retrouvons les mêmes accents tragiques dans l'évocation d'un conflit opposant les membres d'une même famille et inscrivant au centre du poème le thème du sang qui souille car injustement répandu ${ }^{38}$ : "nos mains et nos glaives (sont) ruisselants du sang de nos frères" ${ }^{\prime 39}$.

19 Enfin, la pensée du chef de file du mouvement humaniste français, Nicolas de Clamanges, est la plus complexe car appuyée à la fois sur les Ecritures et l'histoire romaine. Dans l'Expositio super Isayam, il cherche à prouver que la France est l'Egypte décrite par le prophète Isaïe. C'est alors Virgile qui vient à l'appui de sa démonstration, ce qui n'est pas étonnant car l'Enéide ${ }^{40}$ est un de ses ouvrages de référence, qu'il cite fréquemment. Comme Chartier ou Premierfait, il a également en tête les épisodes des guerres civiles romaines, mais eu égard à son sujet, il choisit celles qui ont eu sur le théâtre égyptien, 
c'est-à-dire la guerre entre Marc-Antoine et Octave ${ }^{41}$. Toutefois, des trois humanistes, c'est lui qui développe le moins ce thème à la lumière de l'histoire romaine, préférant le recours aux auteurs prophétiques. Ces descriptions ne sont toutefois pas uniquement destinées à montrer l'étendue des connaissances historiques des humanistes mais bien à ajuster et à réactualiser les notions de roi et de tyran, au regard des événements du début du XVe siècle.

\section{Distinguer le bon prince du tyran au $\mathrm{XV}^{\mathrm{e}}$ siècle}

Jean Petit le premier actualise la définition du tyran en présentant Louis d'Orléans comme un être avide, qui rêvait d'être roi à la place de son frère. Le théologien passe à dessein sur le fait qu'à cause des crises de folie de Charles, Louis exerçait souvent la réalité du pouvoir. En outre, les mêmes reproches auraient pu être adressés à Jean sans Peur. Les humanistes s'attachent alors à mettre en évidence les traits de caractère qui définissent à coup sûr le tyran et l'opposent au roi juste. Chartier, on s'en souvient, méprise visiblement Sylla, dont il dénonce le comportement instable lorsqu'il évoque sa rage, non pas avec un terme suggérant la colère comme ira mais grâce à rabies qui souligne une fureur incontrôlable comparable à la démence ${ }^{42}$. Cette tournure fait d'ailleurs écho à celle utilisée par Pierre d'Ailly en 1419, dans une lettre à Gerson et dans laquelle il parle de la "rage du lion" rabies leonis, en rappelant les massacres parisiens de $1418^{43}$. La rage est la marque du tyran, on s'en souvient. Il fait peu de doute que Jean sans Peur incarne ce lion enragé. Laurent de Premierfait, dans son $1^{\mathrm{er}}$ prologue des Cas des nobles hommes et femmes, affirme que: "aux nobles principalement affert avoir clemence qui met equitté devant vigueur et veult plus encliner a merci que a vengence sanz saillir hors des termes de justice sanz laquele roys ne roys ne roannes ains sont tirans cruelz et tirannes" ${ }^{44}$.

Sans le binôme Justice et Pitié, "un roy n'est pas roy ains larron" et son royaume est une "larroniere" ${ }^{45}$. Cette tirade, assez véhémente n'est pas sans rappeler les accents de Clamanges dans son traité sur la justice, datant du début des années 1420. D'abord destiné à Louis de Guyenne, l'auteur le dédicace, après la mort de ce dernier, au duc Philippe de Bourgogne, fils de Jean sans Peur.

"Il est notoire que le roi se distingue du tyran par la justice: alors que le roi, par l'exercice de la justice préserve les droits et les libertés du peuple, le tyran au contraire use de l'injustice pour l'opprimer"46, "[...] que la justice provoque le pardon et que la clémence tempère la rigueur de la justice $e^{47}$. [...] La patrie dans laquelle il est [...] permis au prince d'extorquer à ses sujets ce qu'il voudra, selon son bon plaisir [...] n'est rien d'autre qu'un repère de brigands" ${ }^{88}$.

Et Chartier d'insister: "Bon roi, [...] dont la patience l'emporte sur la démence, la clémence sur la témérité, car [...] la mansuétude et la bonté ont été domestiquées par l'usage [...." ${ }^{49}$. Le bon ${ }^{50}$ roi est celui qui sait user du pardon pour faire régner la justice et mettre fin à la folie meurtrière de la guerre civile. L'aveu de Jean sans Peur et l'exercice de la justice royale sont nécessaires à l'extinction du cercle vicieux de la vengeance. Comme le dit Clamanges: "il ne peut y avoir de vraie paix sans justice" ${ }^{51}$.

Par ailleurs, dans l'Expositio, Clamanges donne au terme "tyran" une définition différente de celle de Jean Petit: c'est un homme qui exerce le pouvoir ${ }^{52}$ et ne se contente pas d'en rêver. A partir du XIV e siècle, la théorie d'une élection divine des princes de France se répand dans le royaume. Laurent de Premierfait n'est pas resté insensible à cette 
"propagande" puisque dans son prologue à la traduction du De amicitia, dédicacé à Louis de Bourbon, il évoque: "la lignee royalle seignouriant en France, dont le glorieux nom est magnisfié et congneu par les 7 climats du monde [...] par le don de Dieu"53.

C'est l'époque à laquelle Jean de Montreuil réactualise la loi salique pour justifier le transfert du trône de France à la branche Valois plutôt qu'au roi d'Angleterre. Et Chartier, proche et fidèle du futur Charles VII reprend à son compte cette idée de l'origine divine du pouvoir royal en France ${ }^{54}$. Selon lui, le royaume de France est une terre bénie, chère à Dieu $^{55}$ : le sang du martyr saint Denis au III ${ }^{\mathrm{e}}$ siècle consacre la France au rang de pays d'élection du christianisme. Chartier ajoute ainsi sa pierre à l'édifice, en construction, de la France championne du christianisme. Enfin, le peuple de France a pour patronne nulle autre que la Vierge Marie, mère de Dieu: Virgo puerpera, [...] Dei genitricem, gallice gentis patronam $^{56}$, ce qui renforce la symbolique de la maison des fleurs de lis.

Chartier est favorable au gouvernement d'un seul homme à l'aura divine, c'est le principe de la monarchie française, dont il est un fervent défenseur. L'action de Marius, créateur d'une armée suffisamment puissante et disciplinée pour être victorieuse des ennemis divers et surtout d'une nouvelle figure dans le paysage politique romain, celui de l'homme providentiel, protégé des dieux et admiré de la population qui le vénère, rencontre probablement aussi son approbation. L'existence d'une armée de métier, tout comme l'établissement d'impôts, est nécessaire à la création d'un Etat fort. Le clan bourguignon refuse cette mutation et son appel à la réforme est d'abord un refus des mutations de l'Etat, appuyées au contraire par Louis d'Orléans puis par les partisans de sa politique. La comparaison de Jean sans Peur à Sylla, défenseur de la république aristocratique, trouve ainsi son explication. Clamanges enfin, décrit la France, auparavant la plus admirable ${ }^{57}$, comme un royaume occupé, depuis que le roi d'Angleterre Henri V s'en est emparé. Mais ce "valeureux chef de guerre" selon Clamanges, est mort ${ }^{58}$, après s'être comporté en tyran, contrairement à ce qu'il promettait d'être grâce à ses qualités. Dans les paroles de l'humaniste perce toutefois l'admiration qu'il éprouvait pour le roi défunt, mais également une déception d'autant plus forte qu'il avait un temps placé ses espoirs en Henri $\mathrm{V}^{59}$. Cette chute rappelle celle de Marius, décrite par Laurent de Premierfait, on s'en souvient. Cependant, le traité de Troyes, signé en 1420 livre le royaume aux Anglais, "transférant le gouvernement aux ennemis"60.

Un peu plus loin, par une jolie synecdoque, Clamanges se fait le témoin de l'attachement de l'opinion à cette transmission de la fonction royale, dont la couronne est le symbole, aussi bien parmi les "intellectuels" dont l'humaniste fait partie, que dans le peuple en général: après le traité de Troyes, "le royaume est occupé par les ennemis, le sang royal rejeté au loin ${ }^{61 “}$. Le Dauphin est effectivement évincé de la succession au trône. Plus loin, Clamanges souligne que c'est la "couronne" qui est passée entre d'autres mains ${ }^{62}$; elle représente certes la fonction, mais pas la "personne" du roi, dont la qualité royale est donc invariablement transmise biologiquement.

L'exercice du pouvoir ne suffit donc pas à faire le roi, la légitimité du gouvernant dépend en grande partie de son ascendance et de l'origine divine de ce pouvoir, rejetant ainsi l'usurpateur dans la catégorie tyrannique. Peut-on alors de bon droit se débarrasser du tyran? Les choses ne sont pas si simples. "Je livrerai l'Egypte entre les mains d'un maître sévère et un roi cruel les dominera ${ }^{63}$. Ce qui est confirmé par une autre citation: par moi les rois règnent et les tyrans occupent la terre ${ }^{64 "}$. Clamanges annonce ainsi dans l'Expositio le commentaire d'Isaie 19:4, qui correspondrait à la situation vécue par le royaume de France depuis la défaite d'Azincourt. Les Egyptiens représentent métaphoriquement les 
Français, livrés à des maîtres cruels. Pour renforcer son exégèse, Clamanges puise chez saint Augustin ${ }^{65}$, qui se démarque du latin de la Vulgate, en introduisant le concept de tyran à un endroit auquel il ne figurait pas à l'origine. Le choix de Clamanges ne doit bien sûr rien au hasard. Il enchaîne:

"En effet, celui qui est un tyran dans le monde grec, est désigné chez les Latins comme un roi puissant. C'est [Dieu] qui fait régner, non seulement le tyran mais également le fourbe, à cause des péchés du peuple ${ }^{66}$. D'ailleurs, le tyran et le fourbe forment plus souvent une seule et même personne et il est rare qu'un tyran ne soit pas également un fourbe et qu'un fourbe ne tende pas à être un tyran"67.

La tyrannie est ainsi explicitement liée aux péchés capitaux et devient un flagellum Dei. Ce roi puissant, d'aucuns pensent qu'il s'agissait du roi d'Angleterre Henri $V$, envoyé pour dominer l'Egypte-France mais récemment décédé. D'après l'humaniste, il fut fort, valeureux et prudent, puisqu'il mit à profit les troubles de la guerre civile pour conquérir la plus grande partie du royaume de France, dans l'espoir d'y régner ${ }^{68}$.

Résumons: les Français, accablés du poids de leurs vices, se déchirent dans une guerre fratricide; un tyran doit, en guise de punition, leur imposer sa cruelle domination, donc Henri V en réussissant cet exploit est le tyran envoyé par Dieu pour châtier les Egyptiens métaphoriques, c'est-à-dire les Français, de leurs péchés. La violence et l'injustice du pouvoir tyrannique assument ici une fonction providentielle de châtiment des péchés, comme c'est déjà le cas dans le Policraticus de Jean de Salisbury ${ }^{69}$, que Clamanges a sans doute étudié. Le tyrannicide n'est donc pas envisageable si Dieu lui-même se trouve à l'origine de l'installation du pouvoir tyrannique. Dieu pourvoira au châtiment du tyran, comme l'explique Alain Chartier.

Les tyrans sont d'après lui les fauteurs de guerre, ceux qui en "actionnant des armes

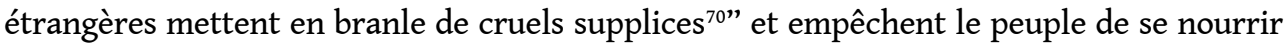
de la paix ${ }^{71}$. La notion de tyrannie chez l'auteur est donc explicitement liée aux guerres, civile et anglaise: le tyran est prêt à tout pour servir ses propres intérêts, y compris à s'allier à des forces extérieures. Mais celui qui rejette la mission de défense des intérêts du peuple, confiée à lui par Dieu en échange de sa place prééminente dans la hiérarchie sociale, s'expose à la colère divine: une vie et une mort, viles, pleines d'opprobre, damnées ${ }^{72}$.

31 Le ton de l'humaniste se fait ensuite presque menaçant: que les Grands prennent garde, s'ils ne remplissent pas leur mission, le peuple qu'ils doivent défendre, sera peut-être tenté de rejeter leur domination, de mettre en doute la légitimité de leur statut social. Alors, le risque sera encore la guerre civile, la subversion de l'ordre établi par Dieu. Ce dernier risquerait bien d'exercer sa colère à leur encontre et de se débarrasser "des racines et des troncs inutiles qui encombrent la terre"73.

Chartier, assez audacieusement, sous-entend que lorsqu'un membre du corps ne remplit plus sa fonction, les autres membres peuvent légitimement contester sa place dans la hiérarchie. Le peuple accepte de servir les Grands et les princes, s'ils le méritent, c'est-àdire s'ils les protègent, les défendent, dans le respect de la justice. Le peuple supporte la "domination naturelle" de ceux qui lui sont supérieurs ${ }^{74}$, à condition qu'ils lui garantissent la paix, la béatitude, sinon, par angoisse, il cède aux sirènes des fausses promesses $^{75}$. Une fois de plus, nous pensons au Policraticus, dans lequel se trouve exposée la théorie du tyrannicide la plus unique par son ton péremptoire. Pour Jean de Salisbury, le prince incarne le respect de la loi et se considère comme le serviteur du peuple qu'il gouverne. Donc, l'autorité souveraine est dépendante de la loi et de la justice. Au 
contraire, le tyran opprime le peuple, donc il peut et doit être tué. La démonstration est également illustrée par la métaphore de l'arbre dont la racine malade doit être coupée, pour la survie de l'ensemble. L'analogie entre ce passage et le poème de Chartier est tellement frappante qu'il semble inconcevable que l'humaniste n'ait pas lu ou au moins eu connaissance de l'œuvre majeure de Jean de Salisbury. Toutefois, la grande différence est que Chartier s'en remet à Dieu et non à la révolte populaire pour châtier le tyran. La morale du poème est en tout cas claire: si l'on veut éviter de nouveaux massacres, chacun doit assumer la mission qui lui a été dévolue par Dieu et favoriser le retour à la paix et au bonheur qui en découle. L'idée d'un consensus entre le souverain et le peuple se retrouve d'ailleurs sous la plume de Premierfait.

L'histoire du roi Roboam, dont une partie des sujets accablés d'impôts se séparent au profit de Jéroboam, est l'occasion d'un commentaire sur le "contrat" liant le roi et ses sujets. Le peuple devient un "conserf" et en contrepartie de son travail, qui donne au prince "toute la force et la seurté", le souverain s'engage à lui "garder le salut et le repoz" ${ }^{76}$. Carla Bozzolo ${ }^{77}$ remarque que cette notion de pacte mutuel n'est pas suggérée par le texte de Boccace. On peut donc en conclure qu'il s'agit bien d'un avis personnel.

Même si elle n'est pas explicite, l'idée de pacte réciproque entre la noblesse, dont le roi fait partie, et le peuple est très claire chez Chartier et paraît donc étonnamment similaire à celle développée par Laurent de Premierfait dans sa traduction de Boccace. Comme dans cet ouvrage, le thème du tyran est intimement lié à celui du consentement populaire, ce qui ne peut être une coïncidence. La défense du peuple est enfin un thème cher à Clamanges, qui dénonce ses souffrances dans le De lapsu et y revient bien plus longuement encore tout au long de l'Expositio, dans laquelle il n'hésite pas à affirmer que la noblesse ne cesse d'opprimer le peuple ${ }^{78}$. Une étude plus approfondie de ce thème permettrait sans doute d'éclairer l'évolution de cette imbrication des notions de patriotisme et de "contrat social" chez les humanistes français de la $1^{\text {ère }}$ et $2^{\text {nde }}$ génération.

Ainsi, les trois auteurs se rejoignent sur la façon globale d'utiliser le concept de "tyran": loin de la définition actualisée de Jean Petit, qui faisait du tyran celui qui rêve du pouvoir et complote pour l'obtenir, les auteurs combinent une définition plus traditionnelle du pouvoir tyrannique à une défense du consensus unissant le peuple à son souverain et à sa classe dirigeante. Celui qui, à l'inverse du bon souverain, gouverne dans son seul intérêt, de façon arbitraire et surtout contraire à l'intérêt de ses sujets, est un tyran. Lorsque le pouvoir est exercé à des fins mauvaises, peccamineuses, il devient un crime, d'autant plus qu'il fait injure à Dieu, en remettant en cause la hiérarchie sociale instaurée par Lui. Toutefois, les humanistes ne légitiment jamais clairement le tyrannicide, préférant appeler de leurs vœux un royaume dans lequel s'exerce la justice et abandonnant à Dieu le châtiment des tyrans.

D'ailleurs, le 10 septembre 1419, le "tyran" Jean sans Peur est assassiné sur le pont de Montereau et le commanditaire, le dauphin Charles, est évincé de la succession au profit du roi étranger, Henri $V$, réduisant à néant les espoirs de paix nourris par Chartier et Clamanges. Cependant, la réaction populaire suscitée par l'intervention de Jeanne d'Arc prouve que les humanistes partageaient avec le peuple de France un sentiment patriotique naissant et surtout la confiance en la légitimité d'une divine lignée de rois ${ }^{79}$. 


\section{BIBLIOGRAPHIE}

Sources

CHARTIER, Alain - Ad destestationem belli gallici et suasionem pacis. Ed. Pascale Bourgain-Hemeryck - Les œuvres latines d'Alain Chartier. Paris: CNRS, 1977, pp. 225-237.

CLAMANGES, Nicolas - Expositio super quadraginta septem capitula Isaye, Arsenal, ms. 137.

CLAMANGES, Nicolas - De lapsu et reparartione justitie, Bâle, 1519.

CLAMANGES, Nicolas - Epistola CXXXVII, Quoniam princeps illustris. Ed. Lydius - Opera Omnia. Leyde, 1613, T.II, pp. 347-353.

MONTREUIL, J. - Opera IV. Monsteroliana. Ed. Ezio Ornato, Gilbert Ouy, Nicole Pons. Paris: CEMI, 1986, épître 202, pp. 278-279.

PREMIERFAIT, Laurent de - Des Cas des nobles hommes et femmes. Paris, BN, fr.131. Ed. Carla Bozzolo, inédit.

PREMIERFAIT, L. - De l'amitié, Paris, BN, fr.24283.

Sources secondaires

BOCCACE - De Casibus, II, 5, “contra filios tyrannorum”. Ed. P.G Ricci et V. Zaccaria. Milan: Arnoldo Mondadori, 1984.

MONSTRELET, E. - Chroniques. Ed. J.A. Buchon. Paris: Verdière, 1827.

PATERCULUS, V. - Histoire romaine, II. Ed. Baptiste Lescaut. Anvers: Au navire d'argent, 1679.

SAINT AUGUSTIN - De Civitate Dei, Livre V, chap. 19. Ed. J.P Migne - Sancti Aurelii Augustini opera omnia, Patrologie latine 32-47. Paris: Ateliers catholiques, 1844, réimp. Turnhout: Brépols, 1993.

Études

AUTRAND, Françoise - Charles VI. Paris: Fayard, 1986.

BOZZOLO, Carla - "L'intérêt pour l'histoire romaine à l'époque de Charles VI: Laurent de Premierfait". in Saint-Denis et la royauté, études offertes à Bernard Guenée. Paris: Publications de la Sorbonne, 1999, pp. 109-124.

BOZZOLO, Carla - Un homme populaire et de petite science au service des hommes de pouvoir: l'humaniste Laurent de Premierfait". in Karen L. Fresco (ed.), Authority of Images / Images of Authority: Shaping Political and Cultural Identities in the Pre-Modern World. Kalamazoo: Western Michigan University, 2016, pp. 159-171.

BOZZOLO, Carla - "La conception du pouvoir chez Laurent de Premierfait". Réimp. in BOZZOLO, Carla (ed. ) - Un traducteur et un humaniste de l'époque de Charles VI: Laurent de Premierfait. Paris: Publications de la Sorbonne, 2004, pp. 53-68

COVILLE, Alfred - Le véritable texte de la justification du duc de Bourgogne par Jean Petit, 8 mars 1408. Paris: Daupeley-Gouverneur, 1911.

COVILLE, Alfred - Jean Petit. La question du tyrannicide au commencement du XV siècle. Paris: Picard, 1932. 
COVILLE, Alfred - "Un ami de Nicolas de Clamanges, Jacques de Nouvion (1372-1411)".

Bibliothèque de l'école des chartes. Paris; Genève: Droz, 1935, tome 96, numéro 1, pp. 63-90.

GAUVARD, Claude - De grace especial. Crime, État et société en France à la fin du Moyen Âge. Paris: Publications de la Sorbonne, 1991.

GAUVARD, Claude - Violence et ordre public au Moyen Âge. Paris: Picard, 2005

GUENÉE, Bernard - Entre l'Eglise et l'Etat. Quatre vies de prélats français à la fin du Moyen Âge. Paris: Gallimard, 1987.

GUENÉE, Bernard - Un meurtre, une société. L'assassinat du duc d'Orléans (23 novembre 1407). Paris: Gallimard, 1992.

LECUPPRE-DESJARDIN, É. - Le royaume inachevé des ducs de Bourgogne (XIVe-XVe siècle). Paris: Belin, 2016.

LEVELEUX-TEXEIRA, Corinne - "Du crime atroce à la qualification impossible. Les débats doctrinaux autour de l'assassinat du duc d'Orléans 1408-1418". in FORONDA, F.; BARRALIS, Ch.; SÈRE, B. (dir. ) - Violences souveraines au Moyen Âge. Travaux d'une école historique. Paris: PUF, 2010, pp. 261-270

LEVELEUX-TEXEIRA, Corinne - "Le lien politique de la fidélité jurée (XIII ${ }^{\mathrm{e}-X V}{ }^{\mathrm{e}}$ siècle)". in ALLIROT, A-H.; GAUDE- FERRAGU, M.; LECUPPRE, G.; LEQUAIN, É.; SCORDIA, L.; VÉRONÈSE, J. (eds.) - Une histoire pour un royaume $\left(X I I^{e}-X V^{e}\right.$ siècle). Actes du colloque Corpus regni en hommage à Colette Beaune. Paris: Perrin, 2010, pp. 197-217.

LEVELEUX-TEXEIRA, Corinne - ; RIBÉMONT, Bernard, (dir. ) - Le crime de l'ombre. Complots, conspirations et conjurations au Moyen Âge. Paris: Klincksieck, 2010.

KRYNEN, Jacques - Idéal du Prince et pouvoir royal en France à la fin du Moyen Âge (1380-1440). Paris: Picard, 1981.

SCHNERB, Bertrand - Les Armagnacs et les Bouguignons. La maudite guerre. Paris: Perrin, 1988.

SCHNERB, Bertrand - L'État bourguignon (1363-1477). Paris: Perrin, 1999.

SCHNERB, Bertrand - Jean sans Peur. Le prince meurtrier. Paris: Payot, 2005.

SWANSON, Robert N. - Universities, Academics and The Great Schism. Cambridge: Cambridge University Press, 1979.

JOLLIVET, Lucie - Les humanistes français face aux crises du début du XVe siècle. Rennes 2, 2013. Thèse de doctorat. Disponible en ligne sur theses.fr.

\section{NOTES}

1. À propos de l'histoire de ces théories, on pourra lire avec profit TURCHETTI, M. - Tyrannie et tyrannicide, de l'Antiquité à nos jours. Paris: PUF, 2001. Quelques années avant le meurtre, en 1400, un des modèles du mouvement humaniste français, le chancelier de Florence Coluccio Salutati, termine son ouvrage De tyranno, dans lequel il pose le débat autour du choix de Dante de mettre les meurtriers de César en Enfer. Le tyran ne peut selon lui être tué que sur l'ordre du pouvoir en place et selon la loi, sinon, il s'agit d'un crime. César exerçait le pouvoir légalement, donc Dante a bien placé Brutus et Cassius. La théorie de Salutati est fort intéressante car elle introduit en outre dans la définition de la tyrannie la notion de péché capital, celui d'orgueil. Les humanistes français s'en inspireront sans doute, nous le verrons. 
2. Concernant l'assassinat et ses conséquences douloureuses, voir GUENÉE, Bernard - Un meurtre, une société. L'assassinat du duc d'Orléans, 23 novembre 1407. Paris: Gallimard, 1992.

3. Dans cet article, seuls seront analysés certains travaux des trois humanistes que sont Nicolas de Clamanges, Laurent de Premierfait et Alain Chartier. D'autres membres de ce milieu, comme Jean Gerson ou Pierre d'Ailly, ne seront évoqués qu'en filigrane, pour des raisons matérielles, non de pertinence.

4. Ils sont tous deux nés dans la décennie 1360. Laurent de Premierfait meurt cependant beaucoup plus tôt, en 1418, sans doute victime de la peste, alors que Clamanges vit jusqu'en 1437.

5. Nous remercions chaleureusement Carla Bozzolo qui a mis à notre disposition ses travaux inédits concernant Laurent de Premierfait et nous a permis d'en faire l'usage dans cet article. Sans sa généreuse contribution, cet article n'aurait pu être aussi complet.

6. BOCCACE - De Casibus. Ed. P.G Ricci et V. Zaccaria. Milan: Arnoldo Mondadori, 1984, II, 5, "contra filios tyrannorum".

7. Voir SCHNERB, B. - Jean sans Peur. Le prince meurtrier. Paris: Payot, 2005.

8. $1^{\mathrm{er}}$ siècle après J.C.

9. "Il est licite de repousser la force par la force". Voir MONSTRELET, E. - Chroniques. Ed. J.A. Buchon. Paris: Verdière, 1827, p. 277 et suivantes, numérisé par Google Books, ainsi que COVILLE, Alfred - Le véritable texte de la justification du duc de Bourgogne par Jean Petit, 8 mars 1408. Paris: Daupeley-Gouverneur, 1911 et COVILLE, Alfred - Jean Petit. La question du tyrannicide au commencement du XV siècle. Paris: Picard, 1932.

10. Voir GUENÉE, Bernard - Entre l'Eglise et l'Etat. Quatre vies de prélats français à la fin du Moyen Âge. Paris: Gallimard, 1987, pp.188-189.

11. Il s'agit d'un autre ami des humanistes Clamanges et Montreuil et appartenant, comme ce dernier, à la maison d'Orléans.

12. Pour plus de détails sur ces événements, voir SWANSON, R. N. - Universities, Academics and The Great Schism. Cambridge: Cambridge University Press, 1979.

13. COVILLE, Alfred - "Un ami de Nicolas de Clamanges, Jacques de Nouvion (1372-1411)". Bibliothèque de l'école des chartes. Paris; Genève: Droz, 1935, tome 96, numéro 1, pp. 63-90.

14. COVILLE, Alfred - "Un ami de Nicolas de Clamanges", p.79.

15. COVILLE, Alfred - "Un ami de Nicolas de Clamanges", p. 80.

16. Voir LECUPPRE-DESJARDIN, É. - Le royaume inachevé des ducs de Bourgogne (XIVe-XVe siècle). Paris: Belin, 2016, chap. 3, "Opportunisme et éthique en politique".

17. MONTREUIL, J. - Opera IV. Monsteroliana. Ed. E. Ornato, G. Ouy, N. Pons. Paris: CEMI, 1986, épître 202, pp. 278-279.

18. CLAMANGES, N. - Expositio super quadraginta septem capitula Isaye, Arsenal, Ms. 137, folio 41, $1^{\text {ère }}$ colonne, "Quid de sepulta, me loqui, attinet justitia, cum latrones atque publicos homicidas, aliorum judices intueri licet, ut verum hodie sit quod propheta dixit?".

19. GUENÉE, Bernard - Un meurtre, une société, pp. 176-179.

20. CHARTIER, A. - Ad detestationem belli gallici et suasionem pacis. Ed. P. Bourgain-Hemeryck - Les cuvres latines d'Alain Chartier. Paris: CNRS, 1977, p. 232, parag. 32, “[...] extrema facinora in publicum emergunt".

21. À ce propos, voir GUENÉE, Bernard - Un meurtre, une société, ainsi que GAUVARD, Claude - De grace especial. Crime, État et société en France à la fin du Moyen Âge. Paris: Publications de la Sorbonne, 1991 et GAUVARD, Claude - Violence et ordre public au Moyen Âge. Paris: Picard, 2005.

22. Concernant les débats autour de ce crime, voir LEVELEUX-TEXEIRA, C. - "Du crime atroce à la qualification impossible. Les débats doctrinaux autour de l'assassinat du duc d'Orléans 1408-1418". in FORONDA, F.; BARRALIS, Ch.; SÈRE, B. (dir.) - Violences souveraines au Moyen Âge. Travaux d'une école historique. Paris: PUF, 2010, ainsi que "Le lien politique de la fidélité jurée (XIII -XV siècle)". in ALLIROT, A-H, et alii (ed.) - Une histoire pour un royaume (XII ${ }^{e}-\mathrm{XV}^{e}$ siècle). Paris: 
Perrin, 2010, pp.197-217. Enfin, LEVELEUX-TEXEIRA, C.; RIBÉMONT, B. (dir.) - Le crime de l'ombre. Complots, conspirations et conjurations au Moyen Âge. Paris: Klincksieck, 2010.

23. CHARTIER, A. - Ad detestationem, p. 232, parag. 32.

24. CLAMANGES, N. - Expositio, fl. 50; Isaïe, 10:1, "Ve qui condunt leges iniquas et scribentem injustitiam scribserunt".

25. CLAMANGES, N. - Expositio, fl. 50, $1^{\text {ère }}$ colonne, "Ecce nominatum contra scribas qui, vel perniciosa principum, assertatione vel spontanea iniquitate, perversa et captiosa, in legum descriptione solent immiscere".

26. CLAMANGES, N. - Expositio, fl. 50, $2^{\text {ème }}$ colonne, "[...] quibus simplices et ignari per subolas irretiantur cavillationes".

27. ClAMANGES, N. - Expositio, fl. 66, "Cuncta siquidem et Ecclesie Dei et regni ipsius illic conflata sunt ac suscitata scandala. An non illic excitata sunt inter principes nostros per gladios acutos ac bicipites flammiferarum linguarum bella civilia que regnum plagis inauditis ad exitium universale conficiunt?".

28. Nous remercions Carla Bozzolo qui nous a fourni "la matière première" de l'analyse qui va suivre en nous confiant une partie de son travail inédit et nous renvoyons à ses articles concernant l'humaniste: "L'intérêt pour l'histoire romaine à l'époque de Charles VI: Laurent de Premierfait". in Saint-Denis et la royauté, études offertes à Bernard Guenée. Paris: Publications de la Sorbonne, 1999, pp. 109-124; "La conception du pouvoir chez Laurent de Premierfait", réimp. in BOZZOLO, Carla (ed.) - Un traducteur et un humaniste de l'époque de Charles VI: Laurent de Premierfait. Paris: Publications de la Sorbonne, 2004, pp. 53-68.

29. BOZZOLO, Carla - "L'intérêt pour l'histoire romaine", p. 120 et suivantes.

30. PREMIERFAIT, L. - Des Cas des nobles hommes et femmes. Paris, B.N. fr.131, fl. 194.

31. PREMIERFAIT, L. - Des Cas des nobles hommes, fl. 200.

32. Voir AUTRAND, F. - Charles VI. Paris: Fayard, 1986, pp. 252-253 et 354-355.

33. PREMIERFAIT, L. - Des Cas des nobles hommes, fl. 202v-203.

34. PREMIERFAIT, L. - Des Cas des nobles hommes, fl. 199.

35. CHARTIER, A. - Ad detestationem, p. 239, parag. 67, "Hanc sillana rabies Rome consecuta est, cujus titulo se pacis auctorem Silla seviens appelavit, cum trucidatis Marianis cujus in conspectu Roma sileret".

36. PATERCULUS, V. - Histoire romaine, II. Ed. Baptiste Lescaut. Anvers: Au navire d'argent, 1679, chapitre XXV, "Putares Sullam venisse in Italiam non belli vindicem, sed pacis auctorem".

37. CHARTIER, A. - Ad detestationem, p. 229, parag. 18, “[...] quid aliud Cesaris et Pompei bello quesitum est, quam romano sanguine campos fluentes defelentibus oculis intueri".

38. A ce propos, voir notre article "Le milieu humaniste français et la réhabilitation de la mémoire de Louis d'Orléans" à paraître dans les actes du colloque en hommage à Nicole Pons, aux Publications de la Sorbonne.

39. CHARTIER, A. - Ad detestationem, p. 233, “[...] excutite ergo, viri, excutite fraterno sanguine manibus madentes gladios".

40. Comme nous l'avons souligné dans notre thèse Les humanistes français face aux crises du début $d u X V^{e}$ siècle, soutenue en décembre 2013 à Rennes 2.

41. CLAMANGES, N. - Expositio, fl. 102, $1^{\text {ère }}$ colonne, "[...] sicut Anthonio et Cleopatra apud actionum bello navali devictis [...] Virgilius introducit".

42. CHARTIER, A. - Ad detestationem, p. 238, parag. 67. 
43. Lettre de Pierre d'Ailly à Gerson, $\mathrm{n}^{\circ} 44 \mathrm{a}$, Glorieux, ed., p. 221, "Hinc scandala multa secuta sunt, hinc potissime superba, rabies leonis in vos aliosque strenuos tunc fidei defensores crudeliter saevientis, quorum plures in seditione parisiensi sunt inhumaniter trucidati".

44. PREMIERFAIT, L. - Des Cas des nobles hommes, fl. $1 \mathrm{v}-5 \mathrm{v}$.

45. PREMIERFAIT, L. - Des Cas des nobles hommes, fl. 267.

46. CLAMANGES, N. - De lapsu et reparatione justiciae libellus. Ed. Johannes de Trittenheim. Bâle, 1519, p. 24.

47. CLAMANGES, N. - De lapsu, p. 3.

48. CLAMANGES, N. - De lapsu, p. 7.

49. CHARTIER, A. - Ad detestationem, p. 239, parag. 71, “Igitur, bone rex, quem [...] patientia vince dementiam, temeritatem clementia reprime. Nam [...] mansuetudinis atque humanitatis usu domantur".

50. Mais le tyran peut également être un prince dissolu et de peu de vertu. Ce point de vue est partagé par les trois humanistes et la critique, acerbe, vise souvent le dauphin Louis de Guyenne, ainsi interpellé par Laurent: "O monstre de homme! O ydole de roy pusilanime qui plus que d'autre doit être fort et magnanime" et méprisé par Clamanges qui le décrit comme "efféminé" et laisse planer le doute sur la rectitude de ses mœurs, puellarum amores, conjugium contemptus. Accusations portées également par le religieux de Saint-Denis et Nicolas de Baye qui soulignent avec perfidie que le jeune homme préfère passer la nuit en compagnie de ses amis plutôt que dans le lit de son épouse...

51. CLAMANGES, N. - De lapsu, p. 19.

52. CLAMANGES, N. - Expositio, fl. 52v, $2^{\mathrm{e}}$ col., "Et super gloriam altitudinis oculorum ejus", hoc est super gloriam superbie ejus que maxime solet in oculis externis elucere. [...] Eum tria sint precipua potencia, sapientia et bonitas. Tyrannus iste superbissimus duo priora sibi attribuit et tertium autem qui pessimus erat, usurpare non presumpsit sed in tercio signo cum magis defecit".

53. PREMIERFAIT, L. - De l'amitié, Paris, BN, fr. 24283, fl. 3v.

54. Sur le prince idéal, voir KRYNEN, J. - Idéal du Prince et pouvoir royal en France à la fin du Moyen Âge (1380-1440). Paris: Picard, 1981.

55. CHARTIER, A. - Ad detestationem, p. 242, parag. 84, "sancti martires Dyonisi cum sociis, quorum sanguine gallica tellus christianae religioni consecrata est".

56. CHARTIER, A. - Ad detestationem, p. 242, parag. 85.

57. CLAMANGES, N. - De lapsu, p. 5, "Ista autem, nisi fallor, causa est ruina et prostrationis hujus regni preclarissimi".

58. CLAMANGES, N. - Expositio, fl. 101, “[...] nonnulli Henricum opinantur, regem anglorum esse recenter exinctum, qui fortis et prudens et strenuus erat et mira valde celeritate propter intestina discidia regni francorum partem ingentissimam occupaverat".

59. En témoigne la lettre 137, "Quoniam princeps illustris" destinée à Henri V en 1417, dans Lydius (ed.) - Opera Omnia, vol II, Epistolae. Leyde, 1613, pp. 347-353. Dans cette lettre, il fait l'éloge des qualités du roi: piété, justice, culture... et souligne qu'il est celui grâce auquel royaume de France retrouvera sa grandeur passée. Il se conduit presque comme un fonctionnaire de l'Etat, fidèle à la res publica plutôt qu'à la personne du roi, donc fidèle à la couronne comme symbole du royaume.

60. CLAMANGES, N. - Expositio, fl. 18, “[...] regnum ipsum ad hostes transtulit”.

61. CLAMANGES, N. - Expositio, fl. 25v, "[...] per hostes occupatio, regii sanguinis eiecto".

62. CLAMANGES, N. - Expositio, fl. 103, “[...]" per corone translationem”. 
63. Isaie, 19:4, "Je livrerai les Egyptiens au pouvoir de maîtres cruels, un roi puissant dominera sur eux".

64. Proverbes, 8:15, "Per me reges regnant et legem conditores justa decernunt", CLAMANGES, N.

-Expositio, fl. 101, $2^{\text {ème }}$ colonne.

65. SAINT AUGUSTIN - De Civitate Dei, Livre V, chap. 19. Dans MIGNE, J.P (ed.) - Sancti Aurelii Augustini opera omnia, Patrologie latine 32-47. Paris: Ateliers catholiques, 1844, réimp. Turnhout: Brépols, 1993, “Per me reges regnant et tyranni per me tenent terram. [...] Apertissime alio loco de Deo dictum est: Quia regnare facit hominem hypocritam propter peruersitatem populi". C'est par moi que les rois règnent. C'est par moi que les tyrans dominent sur la terre. [...] L'Écriture dit ailleurs de Dieu en termes exprès: “C'est lui qui fait régner l'homme fourbe à cause de la perversité du peuple".

66. Job, 34:30: "c'est lui qui fait régner l'homme impie, à cause de la perversité / des péchés du peuple".

67. CLAMANGES, N. - Expositio, fl. 101, 2" col, "Tyrannus enim quod Grecum est, Latine regem fortem notat ipse est, "qui regnare facit", non modo tyrannum, sed etiam ypocritam, "propter peccatam populi", quamquam tyrannus et ypocrita sepius in eandem personam conveniunt et rarus est tyrannus qui non sit ypocrita, rarus insuper ypocrita qui non aliquid de tyranno participet".

68. CLAMANGES, N. - Expositio, fl. 101, $2^{\mathrm{e}}$ col, "Hunc regem fortem, quia Egyptiis Francis dominaturum, dominus ipse pollicetur, nonnulli Henricum opinantur, regem Anglorum esse recenter exinctum, qui fortis et prudens et strenuus erat et mira valde celeritate, propter intestina discidia Regni Francorum, partem ingentissimam occupaverat, ad spem que corona sua estimatione quasi certam venerat". Henri V est décédé à Vincennes en 1422, ce qui nous permet de dater approximativement ces lignes, après la mort du roi. Pour Clamanges, il était le "roi puissant" annoncé par Isaïe.

69. L'édition la plus récente est celle de KEATS-ROHAN, K. - Policraticus I-IV. Turnhout: Brépols, 1993. Nous nous référons quand à nous aux extraits cités par HARF-LANCNER, L. - "Les membres et l'estomac: la fable et son interprétation politique au Moyen Âge". in BOUTET, D.; VERGER, J (ed.) - Penser le pouvoir au Moyen Age, Mélanges Françoise Autrand. Paris: Editions rue d'Ulm, 2000, pp. 111-126.

70. CHARTIER, A. - Ad detestationem, p. 241, parag. 78, "Ubi vero tyrannos extraneaque arma moventes supplicia dura concutiunt".

71. CHARTIER, A. - Ad detestationem, p.241, parag. 80, “Audi vero, miserande populus cujus pax nutrix est et beatitudo tranquillitas".

72. CHARTIER, A. - Ad detestationem, p. 241, parag. 78, “[...] impundenter conturbantius a Deo preter tormentum durissimum, opprobrium vile exitusque vite dampnatissimis [...]".

73. CHARTIER, A. - Ad detestationem, p. 240, parag. 73, "Sed auferet forsitan Dominum pacem vestram et velut truncos inutiles et qui frustra terra occupant radicitus extirpabit".

74. CHARTIER, A. - Ad detestationem, p. 241, parag. 80, “Audi vero, miserande populus [...] excute depulvere servitutis, naturale dominium, cognosce dominium, pacem disce".

75. CHARTIER, A. - Ad detestationem, p. 241, parag. 81, “[...] fortasse dolis malignis et fallacibus promissis seductus angustii incidisti".

76. PREMIERFAIT, L. - Des Cas des nobles hommes, fl. 44r. 
77. BOZZOLO, Carla - "Un homme populaire et de petite science au service des hommes de pouvoir: l'humaniste Laurent de Premierfait", Colloque Images of Authorithy and Authorithy of Images / Images du pouvoir et pouvoir des images. University of Illinois at Urbana-Champaign, 29 avril-1er mai 2002, sous presse.

78. CLAMANGES, N. - Expositio, fl. 101v, $2^{\mathrm{e}}$ colonne, "Cepit nobilitas plebem opprimere". 79. Sur cette question, voir BEAUNE, C. - Naissance de la nation France. Paris: Gallimard, 1985.

\section{RÉSUMÉS}

Le 23 novembre 1407, le meurtre avec préméditation de Louis d'Orléans, frère du roi Charles VI, par les sbires de leur cousin Jean de Bourgogne, remet la question du tyran et du tyrannicide au centre des débats entre intellectuels. En effet, après avoir avoué être le commanditaire du crime, Jean sans Peur revient sur ses déclarations et demande à une équipe de théologiens, dirigée par le normand Jean Petit, de préparer sa défense. Cette justification est exposée au public en 1408 et repose sur un argument repris au juriste-consulte Cassius: vim vi repellere licet. Louis aurait été un tyran dont le meurtre aurait été à la fois licite et juste. L'affirmation apparait comme un écho des débats autour du tyrannicide qui agitent les humanistes italiens depuis la $2^{\text {ème }}$ moitié $d u$ XIV siècle. Mais l'actualité du crime rend la proposition de Jean Petit d'autant plus choquante que la justice royale se trouve paralysée face au refus de l'aveu. Devant l'incapacité de la justice publique à réagir, la famille et les proches du défunt n'ont plus d'autre choix que la vengeance pour obtenir réparation. Le milieu humaniste français, constitué d'humanistes avérés et reconnus, tels Nicolas de Clamanges, Laurent de Premierfait et Alain Chartier, et des universitaires, tentés par ce nouveau mouvement intellectuel, comme Pierre d'Ailly et Jean Gerson, réactivent la polémique italienne en l'adaptant au contexte: Qu'est-ce qu'un tyran? Quelle est la différence entre le tyran et le roi légitime? Est-il licite de tuer le tyran pour sauver le roi? Les humanistes et leurs amis cherchent la réponse la plus juste à ces questions, car l'enjeu est alors immense: la sauvegarde du royaume de France.

On November 23, 1407, the premeditated murder of king Charles' brother Louis d'Orléans by the henchmen of their cousin John of Burgundy puts the question of the tyrant and tyrannicide at the center of intellectual debates. Indeed, having confessed to ordering the crime, Jean sans Peur goes back on his statement and asks a team of theologians led by the Norman Jean Petit to prepare his defense. This justification is exposed to the public in 1408 and is based upon an argument taken to the jurist Cassius: vim vi repellere licet. Louis was a would-be tyrant, so his murder was both lawful and good. This assertion seems to be an echo of the debates surrounding tyranicide raging amoung the Italian humanists for half a century. Due to the refusal of confession, the royal justice is paralized; therefore the Justification by Jean Petit is all the more shocking. Faced with the inability of justice, Louis' family is left with no choice but vengeance. The French humanists milieu, which brings together acknowledged and famous humanists such as Nicolas de Clamanges, Laurent de Premierfait and Alain Chartier, and academics tempted by this new intellectual movement, such as Pierre d'Ailly and Jean Gerson, revive the controversy by adapting it to the context: who can be call a tyrant? What is the difference between a tyrant and a legitimate king? Is it lawfull to kill the tyrant as to save the king? The most appropriate answer 
to these questions must be found because what is at stake is no less than the French kingdom survival.

INDEX

Mots-clés : Humanisme français, tyrannicide, justice, guerre civile, homicide

Keywords : French humanism, tyrannicide, justice, civil war, murder

\section{AUTEUR}

LUCIE JOLLIVET

Université Rennes 2, Tempora (EA 7468), UFR Sciences Sociales, 24307-5043, Rennes, França lucie.jollivet@gmail.com 